\title{
Reflexiones sobre la intervención profesional en el debate contemporáneo argentino
}

\section{Reflections on professional intervention in the contemporary Argentine debate}

\author{
Federico Guzmán Ramonda ${ }^{a}$
}

\begin{abstract}
Resumen
El artículo realiza un conjunto de reflexiones sobre el debate contemporáneo de la intervención profesional en argentina. El mismo expresa una reflexión, una lectura, entre otras posibles que no pretende ser conclusiva pero si resaltar aspectos que considero salientes del mismo.
\end{abstract}

Palabras clave: intervención profesional, comprensión compleja, matrices de pensamiento.

\begin{abstract}
Abstrac
The article makes a set of reflections on the contemporary debate of professional intervention in Argentina. The same expresses a reflection, a reading, among others possible that does not pretend to be conclusive but to highlight aspects that I consider salient of the same.
\end{abstract}

Keywords: profesional intervention, complex comprehension, matrices of thought.

\section{Introducción: debates profesionales y lógica particular de proceso}

En artículo expresa un conjunto de reflexiones sobre el debate acerca de la intervención profesional, para esto considero necesario colocar algunas cuestiones centrales que nos introducen en la lógicas particulares de proceso $^{1}$ que constituyen la particularidad del proceso argentino donde emerge esta conceptualización.

Considero importante abordar el concepto de

1 Cada época histórica nos exige comprender cuáles son las maneras particulares en que los acontecimientos económicos, políticos, culturales se relacionan dentro de determinados campos de posibilidad, o sea, conocer las "lógicas particulares de proceso" dentro de un campo de fuerzas y posibilidades alternativas (Thompson, 1981, p. 117).
Kera Yvoty: reflexiones sobre la cuestión social. Vol. 1, 2016, 21-30.

ISSN (impreso): 2519-7797

a Universidad Nacional del Litoral, Argentina.

Universidad Nacional de

Entre Ríos, Argentina.

Correspondencia a:

Federicorgr@gmail.com

Cita:

Guzmán Ramonda, F.

(2016). Reflexiones sobre la intervención profesional en el debate contemporáneo argentino. Kera Yvoty: reflexiones sobre la cuestión social, 1, 21-30.

Recibido:

5 octubre 2016

Aceptado:

29 noviembre 2016 
intervención desde la óptica de los debates profesionales ya que esta perspectiva brinda una llave de ingreso para comprender esta conceptualización como una construcción plural y situada.

La noción de debate, como expresa, Cazzaniga se refiere al "conjunto de temas que se tornan problemáticas teóricas que despiertan la atención y que desatan análisis, reflexiones e investigaciones transformándose en 'nudos fuertes', sobre los cuales existen disputas a partir de diferencias epistemológicas, teóricas, ideológicas y políticas" (Cazzaniga, 2012, p. 5) ${ }^{2}$. Estos representan "una de las expresiones en que se manifiesta el movimiento histórico de la profesión en el marco de las determinaciones sociales, económicas, políticas y culturales de la sociedad", estos dan cuenta del proceso de interlocución entre los profesionales sobre las formas de ser y deber ser del Trabajo Social en relación con la multiplicidad de aspectos que la profesión tiene (Siede, 2006, p. 47).

Estos debates construidos en el marco de la trayectoria disciplinar, se articulan a una trama mayor, su contexto de producción, de modo que condensan el momento histórico, como también expresan las diferentes tradiciones, culturas y corrientes hegemónicas de pensamiento vigentes dentro de la profesión. Es en el territorio de esta pluralidad conflictiva, constitutiva de la vida académica y profesional, desde donde se afrontan los desafíos que cada coyuntura histórica impone al trabajo socials.

2 Estos debates se pueden identificar a partir de diferentes referencias empíricas donde los mismos toman cuerpo, como son: las producciones escritas (libros, revistas), los temas convocantes de los eventos profesionales, los documentos institucionales, los planes de estudio, los informes finales de las investigaciones, las tesis de grado y pos grado. Estos materiales, entre otros, son los que más nos acercan a tomar contacto con los mismos (Cazzaniga, 2012, p. 5).

3 Pienso la relación entre trabajo social, contexto e historia a partir de las siguientes reflexiones: a) la profesión es socialmente determinada por
Reconocemos que el debate contemporáneo, sobre la intervención profesional, tiene sus comienzos a partir de un movimiento intelectual que la historiografía profesional nombra como pos-reconceptualización. Este constituyo un proceso de búsqueda, debate y crítica que se desarrolla en el trabajo social latinoamericano en general y el argentino en particular, en el marco de los desafíos que ofrecía una nueva coyuntura socio-histórica $(80,90)$. Este movimiento de gran revisión constituye un momento de frontera, donde se desarrollaran rupturas y continuidades con el proceso de reconceptualizador. Su emergencia se encuentra en los cambios del nuevo escenario históricos, la crisis del liberacionismo latinoamericano ${ }^{4}$ y el proceso de construcción teórico política que desarrolla el trabajo social crítico.

Recuperando a Cazzaniga (2009) Podemos considerar a la intervención profesional como la puesta en "acto" de un trabajo o acciones a partir de una demanda que esa "puesta en acto" se configura desde una matriz teórica. El "trabajo profesional", al que entiende "tanto como lugar donde se juega una visión teórica-ideológica específica, como un espacio de acción-consentido sobre la realidad". Son justamente estos dos aspectos -lo teórico ideológico y el sentido- sobre los cuáles nos debemos una vigilancia constante o en todo caso

\footnotetext{
las circunstancias sociales objetivas, las cuales le confieren una dirección social predominante a la práctica profesional, pero también es producto de los sujetos que la construyen colectivamente, en condiciones sociales determinadas (Iamamoto, 1992); b) la profesión se constituye como producto histórico, adquiriendo sentido e inteligibilidad en la historia de la sociedad de la cual es parte y expresión. Los procesos históricos marcan condicionamientos y límites al ejercicio profesional, conformando el terreno sobre el cual se gestan las alternativas de actuación, que "no se traducen de modo mecánico en el ámbito profesional, sino que se encuentran sujetas a innúmeras mediaciones, que deben ser apropiadas y elaboradas por los agentes profesionales tanto en el campo de la producción intelectual como de las estrategias de acción" (Iamamoto, 2003, p. 223).

4 Profundizar Parisí et al. (2012).
} 
un trabajo de elucidación (pensar lo que hacemos y saber que pensamos) en relación a la visión teórica ideológica que poseemos como trabajadores sociales y como ésta se materializa en esa acción con sentido.

Podemos decir de modo general que en nuestro país quien impulsara este debate contemporáneo sobre la intervención será la tendencia teórico-política, que contiene gran pluralidad, que en los últimos 20 años piensa al Trabajo Social desde la perspectiva de Construcción Disciplinar, en la que el énfasis está puesto en lograr mayor conocimiento que posibiliten una comprensión social compleja de la realidad, de las problemáticas sociales, plasmada en una Intervención Social fundada, innovadora (Rozas \& Ludi, 2009)5.

\section{Criticaala perspectiva tecnológica. Re-significación que plantea el debate contemporáneo}

Podemos decir que esta conceptualización contemporánea sobre la intervención profesional se funda en la crítica a la perspectiva tecnológica dentro del trabajo social latinoamericano (Matus Sepúlveda, 2012) que represento una propuesta hegemónica para comprender la intervención profesional, expreso una lógica epistemológica para plantear la relación entre teoría social y practica, como una visión de la profesión en el concierto de las ciencias sociales.

En esta perspectiva el objeto del trabajo social es intervenir aplicando "conocimientos de las ciencias sociales a problemas prácticos" siendo uno de los "objetivos del trabajo social la transformación social”, que se beneficia "de utilizar el método científico" que le

5 El Trabajo Social, como toda disciplina de las ciencias sociales, estructura su campo particular en relación con la intervención profesional y la producción de conocimientos en diálogo con la realidad social. Todo campo disciplinar se construye en torno a problemas e interrogantes acerca de ciertos aspectos de la realidad que se pretende comprenderexplicar-denominar-transformar (Cazzaniga, 2007). otorgaría "eficacia racional a esas acciones de modificación". A su vez, la aplicación del "método científico a los problemas prácticos" sería la fuente generadora de teorías. La autora llama a esta propuesta "modelo de intervención lineal donde existe una evolución que parte del diagnóstico, programa, ejecuta y evalúa” (Matus Sepúlveda, 2012, p. 101).

Se consolida así una "visión instrumental de la metodología”, que reduce la intervención centrando la preocupación en las competencias técnicas, desligándose de la reflexión y preocupación sobre los supuestos existentes en ella, como de las lecturas del contexto, abriendo el camino para una perspectiva a-histórica (Matus Sepúlveda, 2012, p. 27) ${ }^{6}$. A esta propuesta se la denominó "formalismo metodológico" ya que la metodología se cristaliza, tornándose rígida, perdiendo la intervención fidelidad con las particularidades de las situaciones donde se concreta. Además expresa una perspectiva comprensiva centrada en una "matriz instrumental" donde la relación teoría y práctica se expresa en términos de aplicabilidad (Cazzaniga, 2007, p. 27), esta epistemología del metodologismo funda una falsa dicotomía entre conocer y hacer como discursos cerrados que dialogan en sus bordes y no como prácticas mutuamente constitutivas.

La crítica a esta perspectiva colocara su eje sobre dos cuestiones centrales de la misma como son: la matriz instrumental que construye una falsa dicotomía entre el pensar y el hacer, y el formalismo metodológico o metodologismo ${ }^{7}$. Mencionaremos brevemente algunas reflexiones de tres propuestas teóricas que el debate profesional argentino toma para esta crítica y protagonizan, entre otros, el debate contemporáneo sobre la

\footnotetext{
6 En esta perspectiva la intervención se reduce o circunscribe a lo metodológico, se reduce al cómo hacer, sin preguntarse en el fondo por el qué, cómo, y para qué de la misma (Cazzaniga, De Jong, Ludi, 2003).

7 Lima \& Rodríguez (1977).
} 
intervención profesional en nuestro país: la intervención como campo problemático de Rozas Pagaza (2001), la intervención como trabajo de lamamoto (2003) y la idea fuerza de comprensión social compleja que se propone Matus Sepúlveda (2012) ${ }^{8}$.

En el planteo de Rozas Pagaza (2001), comprender los fundamentos y significados de la intervención implica reconocer que ésta institucionaliza y legitima, efectiviza y concreta en el marco de la institucionalidad que el Estado desarrolla para abordar la Cuestión Social (Rozas Pagaza, 2001, p. 22). La propuesta de intervención como campo problemático para captar "los procesos generales que adquieren particularidad en tanto están referidos a las manifestaciones de la cuestión social, que se expresa en la práctica cotidiana de los sujetos" (Rozas Pagaza, 2001, p. 234).

En esta perspectiva teórica para comprender la intervención

no existe una lógica interna que se formalice en una metodología de intervención o en la formalización de modelos de intervención que puedan ser aplicados; su efectividad no depende de la adecuada aplicación de dichas teorías [] en la medida en que existe una apropiación fiel de la realidad se pueden establecer procedimientos operativos que faciliten la intervención profesional [] la preocupación por racionalizar o normativizar la intervención a partir de metodologías formalizadas sin el contexto de aplicación no aporta a la comprensión del campo problemático. (Rozas Pagaza, 2001, p. 114)

Se explicita la relevancia y el lugar que toma la producción de conocimiento, donde el énfasis no recae en lo tecnológico o en la formalización de modelos sino

8 De ningún modo son los únicos autores que realizan esta crítica en el debate profesional argentino, pero constituyen referencias ineludibles del mismo. que la intervención se constituya a partir de una comprensión crítica e histórica de la cuestión social. En síntesis, la noción de campo problemático opera como una mediación conceptual para reconstruir como la dinámica de la cuestión social se torna particular, a partir de la diversa institucionalidad social del estado que se desarrolla históricamente construyendo problemas sociales particulares, donde se pone en acto la intervención profesional.

Iamamoto con su propuesta de conceptuar la profesión como trabajo interpela con esas perspectivas donde el ejercicio profesional aparece como una práctica, constituida que se articula con factores que serían externos, que "inciden" o "condicionan" esa práctica. Este tipo de discursos enuncia que existen "condicionantes internos" y "externos" constitutivos de la intervención: los primeros hacen referencia a competencias profesionales como "accionar estrategias y tácticas, la capacidad de lecturas de la realidad coyuntural, la habilidad en el trato de las relaciones humanas, la convivencia en un equipo profesional"; los segundos serían los que no dependen exclusivamente del agente profesional, como las relaciones de poder institucional, los recursos que son colocados a disposición para el trabajo por la institución que lo contrata, las políticas sociales específicas, etc. (Iamamoto, 2003, p. 94). El trabajo social como fuerza de trabajo cualificada necesita para efectivizarse de medios de trabajo, de condiciones que organizan el proceso de trabajo, estas las provee el empleador (ej. Estado y sus políticas públicas) tiene una relación deasalariamiento con la institución empleadora. El cambio de concepción que implica pasar del enfoque de la practica al trabajo, se expresa en superar una propuesta donde la intervención se funda a partir de una visión dicotómica, dual, entre una dimensión interna y otra externa que permite pensar que la práctica profesional se puede constituir a priori o por fuera de las condiciones contextuales. 
La perspectiva del trabajo construye una mirada que re-articula la intervención profesional constituyéndose a partir de las mediaciones de la coyuntura socio política, como de las condiciones institucionales, considerándose estas elementos constitutivos de del ejercicio profesional.

La propuesta de Matus Sepúlveda propone un giro en la concepción de Trabajo Social que consiste en sacarlo del planteamiento binario hacer/conocer, donde en posiciones extremas el Trabajo Social es una forma de intervención y esta última es acotada como un hacer reflexivo. Expresa que toda intervención es construida a partir de un lugar teórico, a partir de un modo de ver, consecuentemente, no hay intervención sin interpretación social. En el Trabajo Social constituye hay una relación mediada insustituible entre intervención y un sistema de comprensión (Matus Sepúlveda, 2012, p. 27).

$\mathrm{Su}$ propuesta de comprensión social compleja articula las siguientes dimensiones: "los cambios existentes en el contexto, las teorías sociales, los enfoques epistemológicos y los marcos ético/ valóricos" (Matus Sepúlveda, 2012, p. 27). Expresando la historicidad de las formas de compresión (conceptos, categorías, modos de nombrar) e intervención, siendo necesario pensar al trabajo social situado en los procesos sociales donde se concreta, lo que requiere un permanente trabajo sobre el concepto. Manifiesta preocupación en las categorías de comprensión expresando que existe un "prejuicio positivista instalado al suponer que existe una independencia epistemológica entre de los hechos con respecto de las teorías, cuya función sería aprehender descriptivamente estos y sus relaciones" (Matus Sepúlvedda, 2012, p. 48).

Por este motivo, en todo problema social contemporáneo no es posible "partir de la existencia de categorías a priori de las cuales partir incuestionablemente"9. Construir lecturas que recuperen una comprensión compleja supone reconocer "la no existencia de una versión objetiva de lo real. Esto involucra adentrarse en diversos procesos de interpretación, en los choques de racionalidades existentes en todo fenómeno social. Ser racional hoy no significa la posibilidad de existencia de una regla externa de la cual sea posible deducir (...) el criterio con el que proceder" (Matus Sepúlveda, 2012, p. 65). Recuperar las diversas racionalidades existentes en todo fenómeno social y no trabajar con a categorías a priori, exige que la intervención profesional desarrolle una función mediadora que significa reconstruir, recuperando las múltiples voces, discursos, racionalidades y contradicciones puestas en juego en la intervención.

El esfuerzo de la autora se centra en potenciar al trabajo social como una forma de trabajo reflexivo y crítico, que se constituya en una actividad creadora y no en una mera actividad reproductiva, donde desde una comprensión compleja habilita una intervención fundada, ya que no existe modo eficaz de trabajar los social sin nombrar polifónica y reconstructivamente lo social, de esta manera la comprensión compleja entiende la teoría como posibilidad de iluminar contradicciones y "encontrar los caminos para el concreto pensado, para develar las determinaciones múltiple de lo real" (Matus Sepúlveda, 2012, p. 67).

\section{Dimensiones constitutivas de la intervención profesional}

Las reflexiones enunciadas critican

\footnotetext{
9 El trabajo social debe partir cuestionando las categoría con la cual se va a trabajar, el trabajo social debe "profundizar en los fundamentos que hacen surgir esos enunciados" ya que no sólo se van a expresar en una comprensión del problema, sino que se traducirán en diferentes formas de intervención. Este trabajo es necesario para superar el problema de abordar realidades nuevas desde viejos códigos de interpretación (Matus Sepúlveda, 2012, p. 65).
} 
la perspectiva tecnológica del trabajo social, tanto el formalismo metodológico como la matriz instrumental de pensar teoría y práctica. A partir de estas críticas se reconocerán dimensiones constitutivas de la intervención profesional. Se reconoce que la intervención tiene dimensiones constitutivas de toda acción profesional, o sea dimensiones inalienables, presentes en toda intervención: la dimensión teórico-ideológica, la política, la ética y técnico-instrumental. Dimensiones que no se relacionan de modo secuencial ni su articulación es lineal que desarrollaremos brevemente.

Podemos decir que la dimensión teórico-ideológica manifiesta la concepción de mundo, de hombre y de sociedad, a partir de la cual se analiza la realidad social y se fundamenta la intervención. Retomando a Parisí (1993) decimos que el trabajo profesional, se constituye como "un lugar donde se juega una visión teóricaideológica específica, como un espacio de acción-con-sentido sobre la realidad".

La dimensión ética hace refere ncia a la necesidad de la reflexión ética requerida para reconocer las consecuencias que "sobre el otro" produce la intervención (promoción/cancelación de autonomía y derechos) (Cazzaniga, 1996) ${ }^{10}$.

La dimensión técnico-operativa hace

10 Se entiende la intervención como acción con sentido, y ésta se encuentra cargada de valores, normas principios. Por esto es importante pensar que la ética no se agota en el conjunto de principios de "código de ética", sino que al estar las normas, valores y principios implícitos en nuestro obrar, al ser parte constitutiva de nuestra intervención es necesario recuperar la "ética como reflexión" en torno a por qué debemos hacer, que "es la pregunta como reflexión la que permite otorgar las argumentaciones acerca de toda acción, pregunta que a la vez presenta el potencial crítico en el sentido de evaluar, discernir sobre lo establecido o naturalizado" (Cazzaniga, 2006, p. 9). La ética como reflexión intersubjetiva implica una permanente revisión de crítica sobre los sentidos y características de nuestra intervención, de modo que representa una reflexión sobre nuestra ideología, nuestras concepciones o cosmovisiones sobre el mundo y el orden social, puesta en juego en nuestra intervención (Cazzaniga, 2006, p. 9). referenciaal conjuntoarticuladodetécnicas, conocimiento y habilidad del profesional, de modo que es una "categoría relacional" "que se construye en cada momento a partir de las finalidades de la acción, como de los condicionantes del contexto político e institucional". Esta dimensión aborda el conjunto de mediaciones que va desde la concepción a la operacionalización de la intervención (Martinelli et al., 1994, p. 138).

La dimensión política hace referencia a la conflictividad constitutiva de los espacios del ejercicio profesional como las posiciones que frente a estas asume el agente profesional.

No puedo dejar de marcar que en este debatecontemporáneosobrelaintervención profesional emerge y se coloca con fuerte relevancia una concepción epistemológica que apuesta a capturar la tensión teoría/ practica que considera la teoría como caja de herramientas y que sostiene que la producción de conocimientos se da en diálogo con la misma empírea (Cazzaniga, 2007, p. 18) ${ }^{11}$.

\section{Compresión social compleja. Matrices de pensamiento y sentido práctico}

Continuando con la idea de comprensión social compleja, entendemos que el concepto de Matriz teórica nos ilumina, nos brinda elementos para pensar cómo se articulan los modos de comprensión que fundan la intervención. Como expresa Cazzaniga (1996), la "matriz conceptual" representa una "constelación de categorías generales y particulares" que "opera como condición de posibilidad en la construcción de mediaciones conceptuales"

11 La teoría en su propio campo se enfrenta con obstáculos, muros, tropiezos, que hacen que sea relevada por otro tipo de discurso ( ). "La práctica es un conjunto de relevos de un punto teórico a otro, y la teoría, un relevo de una práctica a otra. Ninguna teoría puede desarrollarse sin encontrar una especie de muro y precisa de la práctica para perforarlo" (Foucault, 1995, p. 9). Esta perspectiva epistemológica postula una relación teoría y práctica no en términos de totalización. 
que configuran y construyen el que y sobre que de la intervención.

Para Parisí "nuestra propuesta teórica explicita, siempre por debajo de la misma estamos determinados por un modelo, matriz o paradigma teórico, en el cual -clara o difusamente- estamos inscriptos" (Parisí, 1993, p. 6). De este modo, el "campo categorial en el que efectivamente estemos inscriptos (clara o difusamente) es mediador de nuestra visión de la realidad y el sentido de nuestra práctica profesional" (Parisí, 1993, p. 9).

Estas matrices, al tener sus raíces en procesos socio-históricos concretos, se vertebran de modo multi-causal e influyen en su conformación distintos elementos, como los discursos y propuestas brindadas por la formación académica, la cultura profesional, las culturas institucionales y diferentes racionalidades institucionales, como también lo que Parisí (2007) denomina sentido práctico de la intervención. Este autor introduce un elemento fundamental para comprender la intervención profesional que es la relación, o la dialéctica, de "lo intencional y no intencional" en la construcción del sentido de la intervención. Lo que el autor pone en evidencia es que la construcción de sentido sobre el otro y sobre nuestra intervención, presente en nuestro discursos y nuestras prácticas, no se derivan nunca a priori de la lógica del discurso y la acción consciente, intencionalesyvoluntarias; "porquesiempre pensamos y obramos desde un sentido práctico que parcialmente nos determina" (Parisí, 2007, p. 93). Con "sentido práctico" se refiere a "estructuras no conscientes de ponderación de la realidad internalizadas vía la socialización primaria, básicamente; pero también toda forma de socialización en sentido fuerte (formación profesional por ejemplo) ésta nos determina pero no de modo absoluto" (Parisí, 2007, p. 95).

Esta reflexión se complejiza colocando la necesidad de desarrollar una tarea crítica para esclarecer y comprender cómo se articulan los órdenes de sentido que operan en nuestra acción, el "sentido intencional" y el "sentido práctico" la relación entre ellos no es lineal, puede ser ambiguo y contradictorio y requiere de un proceso de actitud crítica permanente que comienza con el reconocimiento de "descubrirnos determinados" (Parisí, 2007, p. 97).

En este contexto la noción de sentido se torna "algo más" que un objetivo trazado en razón de una planificación de actividades al referirse al entendimiento (propio y ajeno) de los fines últimos que involucra al trabajo profesional. El sentido será deudor de la propia constitución del campo disciplinar y de la inscripción teórica, y a la vez en la misma puesta en acto "producirá efectos sobre sujetos y representaciones sociales" (Cazzaniga, 2009, p. 4).

\section{Intervención en la contradicción. Laautonomía relativa y pensamiento estratégico}

Otro elemento importante que participa de la re-significación del concepto de intervención es la conceptualización del Estado - desde una perspectiva gramscianacomo la "condensación material de las relación de fuerzas sociales" (Poulantzas, 1986). Éste reúne un conjunto de instituciones que condensan y expresan de modo histórico y específico las relaciones de fuerza entre bloques, fracciones y clases sociales. En esta perspectiva, el Estado es una entidad fundamental y estratégica en los procesos de producción y reproducción contradictoria de las relaciones sociales, de modo que no es un "instrumento inerte" en manos de una clase, sino que representa un espacio abierto a las contradicciones de intereses que se expresan en la lucha de clases (Vilas, 1979).

En esta concepción, el Estado no se reduce a la institucionalidad de un gobierno, sino que abarca el conjunto global de prácticas que preservan y reproducen de modo contradictorio las relaciones de hegemonía, donde las políticas públicas y sociales, sus instituciones, contienen de 
modo particular el conflicto social, ya que se organizan como "mediaciones del bloque dominante para desenvolver y consolidar el consenso social necesario a su hegemonía, a su dirección de los procesos sociales" (Faleiros, 1992, p. 9).

Estas conceptualizaciones, a partir de complejizar la lectura sobre el fenómeno de la dominación y la reproducción social, visibilizando el conflicto como constitutivo de las instituciones y sus políticas, abren la posibilidad de desarrollo de autonomía relativa en la dirección del ejercicio profesional por parte de los profesionales. Podemos decir con Cazzaniga que este enfoque crítico permite iluminar la contradicción y "comprender que participamos de los dispositivos de intervención construidos en el escenario capitalista, y por lo tanto la impronta del control social es inherente a los mismos dispositivos como forma de sostener el orden, lo que no debería significar mimetización profesional a los mismos" (Cazzaniga, 2007, p. 90).

Es intentando esa no mimetización que entendemos que Iamamoto brinda una serie de herramientas para pensar la autonomía relativa. Capturando este tema, Iamamoto (1984) expresa que la profesión puede desarrollar autonomía relativa en su ejercicio; la misma no es una cualidad innata de la profesión, sino que encuentra su fundamento en que la intervención profesional reproduce intereses contrapuestos en conflicto, ya que participa al mismo tiempo de los "mecanismos de dominación" como de las estrategias de "sobrevivencia de la clase trabajadora". De este modo, la posibilidad de autonomía relativa en la conducción de la intervención se basa en la existencia de conflicto, que se manifiesta en los contextos institucionales de trabajo, como en su comprensión y la estrategia a desarrollar, ya que "la posibilidad de colocarse en el horizonte de las clases subalternas no anula el carácter contradictorio y conflictivo del ejercicio profesional" (Iamamoto, 1984, p. 96).
Continúa expresando que el sentido o significado social de la profesión no se concreta a partir de la intencionalidad del agente profesional, no hay un sentido sino la condensación de varios intereses o sentidos, racionalidades, etc., que el discurso teórico ideológico del agente profesional y las circunstancias sociales objetivas le confieren direccionalidad a la actividad profesional (Iamamoto, 1984, p. 79). En este sentido, entendemos que la propuesta de autonomía relativa brinda margen y potencialidad para una intervención crítica o con perspectiva emancipatoria pero no anula la "ambigüedad estructural" constitutiva de la intervención que señala Cazzaniga (2007).

Podemos decir que intervención actúa en dos tiempos: la intervención sobre los "problemas particulares", y al mismo tiempo en el campo de los discursos institucionales y sociales acerca de las manifestaciones de la cuestión social, de modo que la intervención es algo más que el trabajo teórico-metodológico que se realiza en situaciones que demandan actuar, ya que "los saberes disciplinares participan en el campo simbólico dirimiendo (o consolidando, depende del posicionamiento ético y político) las construcciones discursivas y las representaciones sociales acerca de las condiciones de vida de los sujetos y las múltiples determinaciones que le dan origen" (Cazzaniga, 2007, p. 91).

Es en este marco donde se instala como fundamental el pensamiento estratégico para pensar la intervención profesional, pensamiento que coloca en su centro una reflexión sobre el poder y como este constituye la intervención profesional. Desde este pensamiento se reflexionara sobre como dialoga la intervención profesional con las dinámicas de la reproducción social, como sobre la influencia de los conflictos sociales en los contextos de intervención profesional y la diversidad de agentes que protagonizan esos conflictos. La reflexión estrategia sobre el carácter del conflicto permite desarrollar 
en el terreno del pensamiento condiciones para construir autonomía en la conducción de la intervención profesional.

Concluyendo podemos decir que en el debate contemporáneo del trabajo social se complejiza nuestra "caja de herramientas" para pensar la intervención profesional. Este proceso se justifica en la necesidad de construir un nuevo arsenal teórico que esté en condiciones de interpretar las nuevas complejidades que el mundo contemporáneo le presenta a la intervención profesional.

\section{Referencias bibliográficas}

Cazzaniga, S. (1996). El Abordaje desde la singularidad. Cuadernillo Temático, 6(22). Recuperado de http://catedras. fsoc.uba.ar/heler/cazzaniga. $\% 20$ el\%2 oabodaje\%2 ode\%2 ola\%2o singularidad.doc

Cazzaniga, S. (2006). Reflexiones sobre la ideología, la ética y la política en Trabajo Social. Presentado en la Reunión de Mesa Nacional de la FAAPSS, PosadasMisiones, Argentina.

Cazzaniga, S. (2007). Hilos y nudos. La formación, la intervención y lo político en el Trabajo Social. Buenos Aires: Espacio.

Cazzaniga, S. (2009). Producción de conocimientos y formación profesional. Algunas consideraciones. In $L a$ investigación en Trabajo Social (vol. 8, pp. 13-22). Universidad Nacional de Entre Ríos. Paraná: Facultad de Trabajo Social.

Cazzaniga, S. (2012). Debates contemporáneos en Trabajo Social. Documento de Trabajo Facultad de Trabajo Social. Manuscrito en preparación.

Cazzaniga, S., De Jong, E. \& Ludi, M. (2003). Fundamentos de la Reforma Curricular. In E. Jong (Ed.), Formación Académica en Trabajo Social. Una apuesta política a repensar la profesión. Buenos Aires: Espacio Editorial.

Faleiros, V. (1992). Trabajo Social e instituciones. Buenos Aires: Humanitas.
Foucault, M. (1995). Un diálogo sobre el poder y otras conversaciones. Madrid: Alianza Materiales.

Iamamoto, M. \& Carvalho, R. (1984). Relaciones sociales y Trabajo Social. Esbozo de una interpretación teórico metodológica. $3^{\text {a }}$ ed. Lima: CELATS.

Iamamoto, M. (1992). Renovação e conservadorismo no Serviço Social. Ensaios críticos. São Paulo: Cortez Editora.

Iamamoto, M. (2003). Serviço Social na contemporaneidade. Trabalho $e$ formação profissional. São Paulo: Cortez.

Lima, L. \& Rodríguez, R. (1997). Metodologismo: estallido de una época. Revista Acción Crítica, (2), 19-48.

Martinelli, M. L. \& Koumrouyan, E. (1994). Um novo olhar para a questão dos instrumentais técnico-operativos. Serviço Social e Sociedade (45), 137-141.

Matus Sepúlveda, T. (2012). Propuestas contemporáneas en Trabajo Social. Hacia una intervención polifónica. Buenos Aires: Espacio Editorial.

Parisí, A. (1993). Paradigmas teóricos e intervención profesional. Revista Conciencia, (1).

Parisí, A. (2007). Sentido práctico, intervención y subjetividad: cinco tesis.In A.A. V. V. (Ed.), Trabajo Social, prácticas universitarias y proyecto profesional crítico. Buenos Aires: Espacio Editorial.

Parisí, A., Peralta, M. \& Videla, V. (2012, Set). La pregunta por la necesidad de una nueva Teoría Crítica. Presentado en XX Seminario Latinoamericano de Escuelas de Trabajo Social, Córdoba, Argentina.

Poulantzas, N. (1985). O Estado, o poder, o socialismo. $\mathbf{2}^{\mathrm{a}}$ ed. Rio de Janeiro: Editorial Graal.

Rozas Pagaza, M. \& Ludi, M. (2009). Algunas reflexiones sobre el trabajo social argentino. Revista Serviço Social e Sociedade, (100), 772-791. 
Rozas Pagaza, M. (2001). La intervención profesional en relación con la cuestión social. El caso del Trabajo Social. Buenos Aires: Espacio Editorial.

Siede, V. (2006). Aportes para la reflexión sobre los debates y proyectos profesionales en el Trabajo Social. In A. A. V. V. (Ed.), Trabajo Social, prácticas universitarias y proyecto profesional crítico. Buenos Aires: Espacio.
Thompson, E. (1981). A miséria da teoria. Ou um planetário de erros. Uma crítica ao pensamento de Althusser. Rio de Janeiro: Zahar.

Vilas, C. (1979). Política social, Trabajo Social y la cuestión social del Estado. Revista Acción Crítica, (6). 